\title{
Anaplastic thyroid carcinoma: review of treatment protocols
}

\author{
Vera Tiedje', Martin Stuschke², Frank Weber ${ }^{3}$, Henning Dralle ${ }^{3}$, Laura Moss ${ }^{4}$ and Dagmar Führer ${ }^{1}$ \\ 'Department of Endocrinology, Diabetology and Metabolism, Endocrine Tumour Center at West German Cancer Center, University Hospital Essen, \\ University of Duisburg-Essen, Essen, Germany \\ 2Department of Radiotherapy, West German Cancer Center, University Hospital Essen, University of Duisburg-Essen, Essen, Germany \\ 3Department of General, Visceral and Transplantation Surgery, Section of Endocrine Surgery, University Hospital Essen, University of Duisburg-Essen, \\ Essen, Germany \\ ${ }^{4}$ Velindre Cancer Centre, Cardiff, Wales, UK
}

Correspondence should be addressed to V Tiedje: vera.tiedje@uk-essen.de

\begin{abstract}
Anaplastic thyroid carcinoma (ATC) is an orphan disease and in most patients fatal. So far no established treatment is available that prolongs survival. Several large retrospective studies have identified negative prognostic markers, analyzed efficacy of multimodal approaches such as radiotherapy with and without concurrent chemotherapy and chemotherapy protocols. Recently, single case reports have suggested some effectiveness of newer therapies targeting single somatic alterations in ATC. Overall, the conclusions that can be drawn from published retrospective studies and the scarce prospective approaches is that new treatment protocols should be developed including surgery, radiotherapy, chemotherapy and targeted therapy approaches and combinational therapy with immunotherapies. These protocols then need to be evaluated prospectively
\end{abstract} to improve ATC patients' outcome in routine care.

\author{
Key Words \\ - anaplastic thyroid \\ carcinoma \\ - treatment protocol \\ - radiotherapy \\ - targeted therapy
}

\section{Introduction}

Anaplastic thyroid carcinoma (ATC) is an orphan disease responsible for most thyroid carcinoma-associated deaths. Incidence is estimated to be 1-2 per million per year (Smallridge et al. 2012).

The histological diagnosis of ATC is challenging. In immunohistochemistry, ATC is characterized by negative staining for typical thyroid markers e.g. thyroglobulin (Molinaro et al. 2017). ATC is per se classified as stage IV disease by the American Joint Committee on Cancer (AJCC) and is further divided in stage IVA (T4a: intrathyroidal ATC and M0); stage IVB (T4b: extrathyroidal ATC and M0) and stage IVC disease (any T and M1) (Smallridge et al. 2012). Initial stage IVC disease is found in $20-40 \%$ of all ATC (Sugitani et al. 2012, Sun et al. 2013, Zivaljevic et al. 2014).
Recent high-throughput sequencing investigations have further elucidated the genetic signature of ATC (Kunstman et al. 2015, Landa et al. 2016, Latteyer et al. 2016a, Tiedje et al. 2017). One important characteristic is a high mutational burden due to the accumulation of different somatic mutations (Landa et al. 2016). Besides TP53 mutations, considered as a genetic hallmark of ATC, several other genetic alterations have been identified to date in ATC e.g. BRAF and RAS mutations that also occur in differentiated thyroid carcinoma (DTC) (Tiedje et al. 2017). Therefore, it is possible that a subset of ATC arises from DTC. Moreover, histology shows that DTC co-exists in around $30-50 \%$ of ATC (Sugitani et al. 2012, Zivaljevic et al. 2014, Tiedje et al. 2017). 
So far, no unified ATC treatment improving overall survival (OS) is established. Several guidelines e.g. from the American Thyroid Association (Smallridge et al. 2012) and the British Thyroid Association (BTA) (Perros et al. 2014) as well as the National Comprehensive Cancer Network and Practical Guidelines for ATC (Haddad et al. 2015) discuss treatment options, including surgery, radioand/or chemotherapy. Recently, in case reports, efficacy of targeted therapy approaches has been reported. Moreover, checkpoint inhibitors might represent new treatment options (Brauner et al. 2016, Latteyer et al. 2016b).

This review will summarize current knowledge on prognostic factors and treatment options for ATC patients.

\section{Prognostic factors and risk stratification}

In multiple retrospective studies analyzing disease course and aiming to identify prognostic factors for ATC, reported OS was highly variable ranging from few weeks to several years (Sugitani et al. 2012, Sun et al. 2013, Zivaljevic et al. 2014). This implies that although ATC is fatal in most cases, risk stratification might be helpful. This would allow the distinction of patients in whom best supportive care should be administered from patients who may be considered for more aggressive treatment, ideally as part of a clinical trial.

Retrospective multicenter studies have shown that ATC survival is stage dependent (Haymart et al. 2013, Wendler et al. 2016). In a study including 699 ATC patients diagnosed between 2003 and 2008, survival, regardless of treatment approach, was 9 months in patients with stage IVA disease, 4.8 months in stage IVB disease and 3 months in stage IVC disease (Haymart et al. 2013).

Negative prognostic markers such as older age, initial distant metastatic disease, large primary tumor diameter $(>5 \mathrm{~cm})$ and elevated white blood count (WBC) have been described in several retrospective studies (Orita et al. 2011, Sugitani et al. 2012, Zivaljevic et al. 2014).

In a recent retrospective Germany multicenter study including 100 ATC patients, pre-therapeutic prognostic factors for longer survival were age $<70$ years, absence of distant metastases and complete local resection (Wendler et al. 2016).

A Japanese group has prospectively evaluated 74 ATC patients using a prognostic index (PI) for treatment stratification. Four characteristics were included in the PI: acute symptoms, tumor size $>5 \mathrm{~cm}$, distant metastasis and $\mathrm{WBC}>10.000 / \mathrm{mm}^{3}$ (max. 4 points). ATC patients with low PI $(\leq 1)$ were treated more aggressively with multimodal treatment (surgery, radiotherapy and chemotherapy) compared to subjects with higher PI. Mean survival of ATC patients with $\mathrm{PI} \leq 1$ was significantly longer (442 days) compared to patients with $\mathrm{PI} \geq 3$ (113 days) (Orita et al. 2011).

In a single-center Chinese study a prognostic score, which comprised age $(<55$ years: score $0, \geq 55$ years: score 1$)$, blood platelet count $\left(<300 \times 10^{9} / \mathrm{L}\right.$ : score $0, \geq 300 \times 10^{9} / \mathrm{L}$ : score 1$)$, WBC $\left(<10 \times 10^{9} / \mathrm{L}\right.$ : score 0 , $\geq 10 \times 10^{9} / \mathrm{L}$ : score 2 ) and stage (IVA: score 0, IVB: score 1 , IVC: Score 2), was evaluated in a cohort of 42 patients. In multivariate analysis, risk group and therapeutic regimen were independent prognostic factors (Sun et al. 2015).

It is discussed whether patients with preceding benign thyroid disease in terms of goiter or differentiated thyroid carcinoma (DTC) that develop ATC may have better prognosis. In a retrospective study analyzing data of ATCs diagnosed in 17 Dutch hospitals from 1989 to 2009, 29 of 94 patients had preexisting goiter $(n=21)$ or DTC $(n=8)$. However, OS was similar in patients with preexistent goiter or DTC (55 days) compared to patients without known previous thyroid disease (56 days) (Steggink et al. 2015).

\section{Surgery}

Due to the nature of the disease in clinical practice, indication for surgery is often highly individualized especially since in many patients ATC is diagnosed after surgery. For instance, data from the American National Cancer Database (NCDB) show that in up to $40 \%$ of patients, the diagnosis was not made prior to surgery (Goffredo et al. 2015). Furthermore, available studies on the role of surgery in the setting of ATC are predominantly of retrospective nature and suffer a selection bias as the multimodal therapy is rather heterogeneous.

One must conclude that available studies are not designed to conclusively proof when and to what extent surgery is warranted in ATC and which patient will truly benefit. However, in general, it is accepted that patients with intrathyroidal and extrathyroidal ATC without invasion of the aerodigestive tract should be discussed for surgery, whereas extrathyroidal ATC with invasion of the aerodigestive tract should not primarily be considered for surgery (Dralle et al. 2013). Similarly, according to the 2012 American Thyroid Association guidelines, surgery in ATC patients is recommended in stage IVA and IVB disease and when gross tumor resection (at least R1) can be achieved (Smallridge et al. 2012).

However, data extraction and analysis from the NCDB show that this recommendation does not reflect the 
clinical reality. Goffredo and coworkers identified a cohort of 680 patients treated between 2003 and 2006. 335 of 680 patients underwent surgery, and stage IVC disease was reported in 25 of these patients (Goffredo et al. 2015). Despite the fact that preoperative resectability must have been assumed, gross tumor resection (i.e. at least R1) was achieved in over $70 \%$ of stage IVB and stage IVC. In contrast, tumor-free resection margins were achieved in almost $60 \%$ in stage IVA disease, while this number drops by half in stage IVB and IVC disease (i.e. $30 \%$ and 36\%, respectively) (Goffredo et al. 2015).

Retrospective studies are prone for selection bias. Based on the NCBD study, when compared to the cohort of patients who did not undergo surgery, any kind of surgery was significantly associated with longer OS in stage IVA (9.7 vs 3.0 months without surgery) and stage IVC patients (3.4 months with vs 1.7 months without surgery) and trend toward significance in stage IVB patients (4.2 with vs 3.4 months without surgery) (Goffredo et al. 2015). The confounding effect might become apparent when we note that in this study, the extent of surgery (i.e. R0 vs R1 vs R2) did not affect the overall survival thus contradicting the general positive effect of surgery (Goffredo et al. 2015).

To this regard, Brignardello and coworkers took a unique approach as they evaluated the role of surgery in advanced ATC (i.e. stage IVB and stage IVC) (Brignardello et al. 2014). This group retrospectively analyzed a cohort of 55 stage IVB and stage IVC patients, treated between 1999 and 2012. Overall, 17 of 24 stage IVB and 24 of 31 stage IVC patients have been found eligible for surgery. In contrast to other studies, the extent of surgery was reported and in addition to thyroidectomy and therapeutic central and lateral neck dissection frequently resection of soft tissue, nerve structures and vascular resection was performed.

Despite their surgical expertise, in 12 of 41 patients (29\%), only 'partial debulking' with gross macroscopic tumor residuum was achieved. Complete tumor resection (R0) was achieved only in 4 patients and microscopic tumor residuum (R1) was reported for 5 patients. Thus, the ATA guidelines were fulfilled only in 9 of 41 patients (21\%). Predominantly, the authors report a 'maximal debulking' $(n=20)$ with minimal macroscopic tumor at vital structure (i.e. aerodigestive tract, carotic artery). The retrospective data analysis suggested that maximal debulking is associated with longer OS (6.5 vs 1.5 months) independent of disease stage. As the postsurgical treatment was very heterogeneous comprising radiotherapy and in some cases chemotherapy and OS analysis was not adjusted for the adjuvant treatment, a confounder cannot be ruled out (Brignardello et al. 2014).

As a consequence of the rarity of this disease, reflected in the existence of multiple retrospective rather than prospective studies and the heterogeneous multimodal management, it is impossible to prove which surgical approach is most beneficial for which patient. Overall, the available literature shows that surgery is clearly warranted in stage IVA disease and when gross tumor resection can be achieved, as overall survival will significantly increase (Goffredo et al. 2015). This recommendation is clearly supported by the only prospective study published so far. De Crevoisier and coworkers report that in a multivariate analysis, gross tumor resection (i.e. R0 or R1) is associated with longer survival (De Crevoisier et al. 2004).

Similar data can be extracted from the Korean nationwide retrospective study in which 329 ATC patients diagnosed between 2000 and 2012 were analyzed. In this study, surgery with curative intent - therefore excluding debulking procedures - was associated with better survival in stage IVA and IVB but not in IVC patients (Baek et al. 2016). Another recent study from Korea shows surgical intervention for selected patients might indeed result in a prolonged survival benefit. Lee and coworkers reported an initial remission rate of $60.5 \%$ and a cure rate of $42 \%$ of patients after initial R0/R1 resection (Lee et al. 2016).

Extent and thus morbidity of surgery must be balanced to the potential benefit. Details on post-surgical morbidity is scarce but was reported by Brignardello and coworkers. This group report recurrent laryngeal nerve injury in 2 of 55 patients (3.6\%) and hypoparathyrodism in 11 of 55 patients (20\%). Two of 55 patients required a

Table 1 Targeted therapy in ATC patients.

\begin{tabular}{ll}
\hline Targeted molecular alteration & Drug \\
\cline { 1 - 1 } BRAFV600E & Dabrafenib + Trametinib \\
& Dabrafenib + Trametinib \\
& Vemurafenib \\
& Vemurafenib \\
ALK translocation & Crizotinib \\
TSC2 truncating mutation (Q1178) & Everolimus \\
\hline
\end{tabular}

Response

ORR $69 \%$

PFS 9 months

PFS 2 months

CR 38 days

$90 \%$ tumor reduction, $\mathrm{PFS}>6$ months

PFS 18 months

\section{Reference}

Subbiah et al. (2017)

Agarwal et al. (2016)

Marten et al. (2015)

Rosove et al. (2013)

Godbert et al. (2015)

Wagle et al. (2014)

ORR, overall response rate; PFS, progression-free survival. 
Table 2 Currently recruiting clinical trials for ATC patients.

\begin{tabular}{l} 
Study title and recruiting countries \\
\hline Atezolizumab Combinations with Chemotherapy for \\
Anaplastic and Poorly Differentiated Thyroid Carcinomas, \\
University of Texas MD Anderson Cancer Center, Texas, USA \\
NCT03181100
\end{tabular}

A Phase II Study of MLN0128 in Metastatic Anaplastic Thyroid Cancer, Dana Farber Cancer Institute, Boston, Massachusetts, USA NCT02244463

Phase II Study Assessing the Efficacy and Safety of Lenvatinib for Anaplastic Thyroid Cancer, (HOPE), Multiple Centers Japan NCT02726503

Phase II Trial of Pembrolizumab in Metastatic or Locally Advanced Anaplastic/Undifferentiated Thyroid Cancer, UT Southwestern Medical Center, Dallas, Texas, USA NCT02688608

Trametinib in Combination With Paclitaxel in the Treatment of Anaplastic Thyroid Cancer, Memorial Sloan Kettering Cancer Center, New York, USA NCT03085056

Phase II Trial of Pembrolizumab in Metastatic or Locally Advanced Anaplastic/Undifferentiated Thyroid Cancer, UT Southwestern Medical Center, Dallas, Texas, USA NCT02688608

An Open-Label, Single-Arm, Multicenter, Phase 2 Trial of Lenvatinib for the Treatment of Anaplastic Thyroid Cancer, Multiple Centers in USA NCT02657369

A Pilot Study of Durvalumab (MEDI4736) with Tremelimumab in Combination With Image Guided Stereotactic Body Radiotherapy (SBRT) in the Treatment of Metastatic Anaplastic Thyroid Cancer, Memorial Sloan Kettering Cancer Center, New York, USA NCT03122496

Ceritinib in Mutation and Oncogene Directed Therapy in Metastatic or Locally Advanced Anaplastic/Undifferentiated Thyroid Cancer, UT Southwestern Medical Center, Texas, USA NCT02289144

A Phase I, Open-label, Multicenter Dose Escalation Study of FAZ053 as Single Agent and in Combination With PDR001 in Adult Patients With Advanced Malignancies, Texas, United States; Ontario, Canada; Tel Aviv, Israel; Koto and Tokyo, Japan; Singapore, Singapore; Barcelona, Spain NCT02936102

A Phase II Trial of CUDC-907 in Patients With Metastatic and Locally Advanced Thyroid Cancer, National Institutes of Health Clinical Center, Bethesda, Maryland, USA NCT03002623

Open-Label Multicenter Phase I/II Study of the Safety and Efficacy of PDR001 Administered to Patients With Advanced Malignancies, United States, Canada, France, Spain, Germany, Hungary, Italy, Lebanon, Netherlands, Poland, Norway, Thailand, Turkey NCT02404441

A Phase II, Open-label, Study in Subjects With BRAF V600EMutated Rare Cancers With Several Histologies to Investigate the Clinical Efficacy and Safety of the Combination Therapy of Dabrafenib and Trametinib, United States, Austria, Belgium, Denmark, Canada, France, Germany, Italy, Korea, Netherlands, Norway and Spain NCT02034110

\section{Drug}

Induction: Paclitaxel

Cohort 1: Vemurafenib,

Cobimetinib + Atezolizumab

Cohort 2:

Cobimetinib + Atezolizumab

Cohort 3:

Bevacizumab + Atezolizumab

Cohort 4:

Paclitaxel + Atezolizumab

MLN0128 (mTor inhibitor)

Lenvatinib

Pembrolizumab

Progression-free survival

Overall survival

Response rate

Trametinib and paclitaxel

Pembrolizumab

Progression-free survival

Response rate

Lenvatinib

Objective response rate

Durvalumab and tremelimumab

Certinib

Progression

FAZ053 (Anti-PD-L1 Antibody) and PDR001 (Anti-PD-1 Antibody)

CUDC-907 (dual inhibitor of HDAC and PI3K signaling)

PDR001 (PD-1 inhibitor)

Dabrafenib and trametinib
Number of participants with Adverse Events (AEs) as a measure of safety and tolerability

Changes in tumor size and metastases

The exposure (AUC (0-336h)) after first dose of treatment Incidence of dose limiting toxicities

Overall response rate

Overall response rate

(Continued)

http://erc.endocrinology-journals.org https://doi.org/10.1530/ERC-17-0435
(C) 2018 Society for Endocrinology Published by Bioscientifica Ltd. Printed in Great Britain 
Table 2 Continued.

\begin{tabular}{l} 
Study title and recruiting countries \\
\hline Phase II Study for the Evaluation of Efficacy of \\
Pembrolizumab (MK-3475) in Patients With Rare Tumors, \\
University of Texas MD Anderson Cancer Center, Texas, USA \\
NCT02721732 \\
An Open-Label, Multicenter, Global Phase 2 Basket Study of \\
Entrectinib for the Treatment of Patients With Locally \\
Advanced or Metastatic Solid Tumors That Harbor \\
NTRK1/2/3, ROS1, or ALK Gene Rearrangements, United \\
States, Australia, Belgium, France, Germany, Hong Kong, \\
Italy, Japan, Korea, Netherlands, Poland, Singapore, Spain, \\
Taiwan and United Kingdom NCT02568267 \\
DART: Dual Anti-CTLA-4 and Anti-PD-1 Blockade in Rare \\
Tumors, National Cancer Institute, USA NCT02834013
\end{tabular}

tracheostoma (3.6\%), and hemorrhage was found in one of 55 patients (1.8\%) (Brignardello et al. 2014). Thus, in the hands of a trained surgeon, the type and rate of surgical complications appear not to be increased compared to the morbidity observed for other thyroid cancer surgeries.

Open questions remain to be solved, such as the timing of surgical and multimodal intervention. Only condensation of these patients in few centers and prospective clinical trials will allow generating the data mandatory. To this end, a recent study by Lai and coworkers from the MD Anderson Cancer center is notable. The group reports of a newly implemented project that allowed to reduce the time between referral to first disposition from 8.7 days to 0.5 days. If this will help to improve overall survival is questionable. However, referral rate has increased and more importantly the rate for enrollment in treatment trials was $34 \%$ which is 10 times higher than the national average for all cancers (Cabanillas et al. 2017).

\section{Radiotherapy}

In a meta-analysis of 17 retrospective studies including 1147 patients it was shown, that radiotherapy improves survival in ATC patients (Kwon et al. 2016). However, it is not certain, as to which cumulative doses and whether radiotherapy vs combined radiochemotherapy before or after surgery is beneficial. A recent large analysis of 1288 patients from the NCDB including patients with

\begin{tabular}{ll} 
Drug & Primary endpoint \\
\hline Pembrolizumab & Non-progression rate
\end{tabular}

Entrectinib

Objective response rate

Ipilimumb and nivolumab Objective response rate non-resected ATC survival was significantly better in patients with radiotherapy with a cumulative dose of $>45 \mathrm{~Gy}$ compared to no radiotherapy or less than $45 \mathrm{~Gy}$ (Pezzi et al. 2017).

A German single-center study analyzed data of 40 ATC patients treated with surgery, radiotherapy and in some cases chemotherapy between 1989 and 2008. Cumulative doses of $50 \mathrm{~Gy}$ or more were associated with better median survival (10.5 months vs 3 months). However, technical approaches varied, e.g. opposing field technique and 3D-conformal technique (Dumke et al. 2014). In another single-center study from China including 60 ATC patients, radiation with cumulative doses of $40 \mathrm{~Gy}$ and higher were also associated with significant better survival (Sun et al. 2015).

In a historic British cohort, data of 20 ATC patients treated between 2004 and 2011 were analyzed. Patients receiving radiotherapy and chemotherapy had significant longer OS ( $n=3$, median 220 days) compared to patients with surgery and radiotherapy ( $n=4$, median OS 176 days) and patients receiving only radiotherapy $(n=6$, median OS 58.5 days). However, no multivariate analysis is provided and the study suffers from its limited number of patients (Lowe et al. 2014).

\section{Chemotherapy}

Clinical studies comparing different chemotherapy regimens in ATC patients are scarce. In a recent German

Table 3 Phase II and III studies of ATC patients.

\begin{tabular}{|c|c|c|c|c|c|}
\hline Study type & Drug & $N$ & Therapy response & Survival & Reference \\
\hline Phase II/III & $\begin{array}{l}\text { C4P } 60 \mathrm{mg} / 2+/- \text { carboplatin } \\
\text { AUC6 + paclitaxel } 200 \mathrm{mg} / \mathrm{m}^{2}\end{array}$ & 80 & & 1-year survival $33.3 \%$ vs $7.7 \%$ & Sosa et al. (2012) \\
\hline Phase II & Sorafenib & 20 & $N=2 \mathrm{PR}$ and $n=5 \mathrm{SD}$ & Median survival 1.9 months & Savvides et al. (2013) \\
\hline Phase II & Pazopanib & 35 & $N=5 \mathrm{PR}$ & OS 19.9 months & Bible et al. (2012) \\
\hline Phase II & Lenvatinib & 17 & PFS 7.4 months & OS 10.6 months & Tahara et al. (2017) \\
\hline
\end{tabular}


multicenter experience including 100 ATC patients treated between 2000 and 2015, 56 patients received any chemotherapy and had significantly better OS compared to those without chemotherapy (Wendler et al. 2016).

Higashiyama and coworkers reported outcome in 13 ATC patients treated with weekly paclitaxel before and after surgery followed by radiotherapy. The response rate was 33\% with one patient achieving complete response, two patients showing partial response and four patients showing stable disease (Higashiyama et al. 2010).

In a case series, 8 ATC patients with stage IVB $(n=4)$ and stage IVC $(n=4)$ disease were treated by surgery and radiotherapy followed by chemotherapy with docetaxel $75 \mathrm{mg} / \mathrm{m}^{2}$ and cisplatin $75 \mathrm{mg} / \mathrm{m}^{2}$ every 4 weeks for 6 courses. The pre-chemotherapy setting differed: two patients had adjuvant chemotherapy with an OS of 3 years and 5.3 years respectively, one patient received induction chemotherapy (OS 3 months) and 5 patients had chemotherapy for distant metastatic disease with an OS of 9 months, 1.7, 4.3, 1 and 3.3 years respectively (Seto et al. 2015).

So far, in the largest placebo-controlled prospective chemotherapy trial for ATC, 80 patients were enrolled. The efficacy of the addition of vascular disruptive agent fosbretabulin (Combretastatin A-4 phosphate) $60 \mathrm{mg} / \mathrm{m}^{2}$ to carboplatin AUC6 and paclitaxel $200 \mathrm{mg} / \mathrm{m}^{2}$ every 3 weeks for 6 cycles was investigated. Patients undergoing surgery $(n=44)$ benefited most from additional fosbretabulin treatment $(n=30)$ with OS of 8.2 months compared to 4.0 months in the control group $(n=14)$. The effect was enhanced by younger age: Patients younger than 60 years who received surgical treatment $(n=23)$ had significantly prolonged OS (10.9 months) when treated additionally with fosbretabulin $(n=17)$ compared to patients only treated with carboplatin and paclitaxel ( $n=6 ; 6.8$ months). In patients who did not undergo surgery OS was similar in both treatment groups (4.0 and 4.6 months) (Sosa et al. 2012). These data suggest that a subgroup of ATC patients may benefit from additional fosbretabulin therapy after surgery. In fact, 1-year survival was longer in patients treated with the combination of carboplatin, paclitaxel and fosbretabulin compared to chemotherapy only (26\% vs $9 \%)$, however, without reaching statistical significance (Sosa et al. 2014).

\section{Multimodal therapy}

Several retrospective studies have demonstrated significant survival benefit for ATC patients treated with a multimodal approach (Haymart et al. 2013, Mohebati et al. 2014, Wendler et al. 2016). In an analysis of the Memorial Sloan Kettering Cancer Center, New York, 95 ATC patients treated between 1985 and 2010 were included. Patients who had surgery, radiotherapy and/or chemotherapy had 42\% 1-year disease-specific survival (DSS) compared to patients without surgery or surgery alone, showing only 6\% 1-year DSS (Mohebati et al. 2014). In contrast, an analysis of 2742 ATC patients diagnosed between 1998 and 2008 (US National Cancer Database) showed only marginal treatment benefit: median survival rates in patients receiving multimodal treatment ranged between 11.2 months in stage IVA patients, 9.9 months in stage IVB patients and 4.9 months in stage IVC patients (Haymart et al. 2013).

\section{Targeted therapy}

The growing knowledge of ATC tumor biology has led to the exploration of targeted therapy approaches. In single case reports, impressive response to BRAF inhibitors vemurafenib (Rosove et al. 2013, Marten \& Gudena 2015, Prager et al. 2016), dabrafenib combined with MEK-Inhibitor trametinib (Agarwal et al. 2016), ALK-inhibitor crizotinib (Godbert et al. 2015) and the mTOR-Inhibitor everolimus (Wagle et al. 2014) were reported. Very recently, a phase II trial including 16 BRAFV600E-positive ATC patients reported an overall response rate of $69 \%$ when patients were treated with the dabrafenib in combination with trametinib (Subbiah et al. 2017). An overview is given in Table 1 . These encouraging results suggest that sequencing to detect potential targets may be a reasonable approach and in addition to ongoing trials (Table 2), subsequent multibasket studies should be initiated.

Apart from these single cases, treatment with multiple tyrosine kinase inhibitors has been explored in ATC patients in several phase II studies with small patient cohorts. In 15 ATC patients treated with pazobanib $(800 \mathrm{mg} /$ day), the median time to progression was 62 days (Bible et al. 2012). In 20 ATC patients treated with sorafenib, 2 patients achieved partial response (PR) and 5 stable disease (SD). The median progression-free survival (PFS) was only 1.9 months (Savvides et al. 2013).

In DTC and PDTC, lenvatinib treatment showed impressive response with even a complete remission (CR) in 4 patients in the SELECT trial (Schlumberger et al. 2015). Recently, a phase II study including 17 ATC patients has shown acceptable treatment response. The OS was 10.6 months and the objective response rate $24 \%$ (Tahara et al. 2017). A more detailed overview is given in Table 3. 
However, since ATCs are characterized by a high mutational burden, the question whether one driver mutation is responsible for disease progression remains open (Landa et al. 2016). Moreover, the field of immunooncology has emerged in the past years and an interaction between somatic mutations and the tumor microenvironment has impressively been shown in several human malignancies (Danilova et al. 2016). Whether combining targeted therapies and immune therapy in ATC patients as suggested for other advanced human malignancies and by in vivo data from preclinical thyroid cancer animal models is an effective approach which needs to be further elucidated (Brauner et al. 2016).

\section{Future perspectives and conclusion}

The treatment of ATC patients is very challenging. ATC is an orphan disease and very aggressive with fatal outcome so far. In addition, in routine care, ATC awareness is lacking. Moreover, no treatment protocols for which improved patient outcome has been demonstrated, are available. Studies evaluating treatment protocols are mostly retrospective, and the investigated populations are very heterogeneous. However, from available data, it may be possible that younger patients with stage IVA and $\mathrm{B}$ disease benefit most from aggressive multimodal treatment.

Often ATC is diagnosed intraoperatively and no precedent scans and endoscopic procedures have been performed to precisely determine local tumor situation. Having a better comprehension would help to decide whether to perform surgery or not and which surgical approach should be chosen. It is still debatable whether patients benefit from debulking operations, due to the high rate of complications. On the other hand, single cases have shown that OS can be improved if aggressive surgery is performed. Prospective studies are needed to clarify the benefit of surgery.

In small cohorts, addition of chemotherapy to radiotherapy has led to an improvement of OS. Moreover, data have shown that at least $40 \mathrm{~Gy}$ should be administered to achieve local tumor control.

Whether chemotherapy or radiochemotherapy improves patient outcome is still unclear. Moreover, whether it should be performed only in stage IVC patients or also in stage IVA and IVB patients remains open. Thus, most reports are retrospective and only very few are prospective in nature. However, since around $80 \%$ patients develop metastatic disease, which often ends fatally, chemotherapy should be administered if possible.
Treatment with new agents targeting driver mutations in ATC has shown effectiveness in single case reports. However, this implies that screening for somatic alterations is done. This is far from being established in routine care.

To improve ATC patient treatment in Germany, a therapy protocol was developed that allows standardized therapy with acceptable toxicity including surgery in selected cases, followed by combined radiochemotherapy and mutational screening of known oncogenes with subsequent targeted therapies (Tiedje et al. 2015).

In conclusion, prospective treatment protocol evaluations are needed and further ATC patients need to be included in multibasket studies including testing for combination of targeted and immune therapies.

\section{Declaration of interest}

The authors declare that there is no conflict of interest that could be perceived as prejudicing the impartiality of this review.

\section{Funding}

This work did not receive any specific grant from any funding agency in the public, commercial, or not-for-profit sector.

\section{References}

Agarwal R, Wang J, Wilson K, Barrett W \& Morris JC 2016 Response to targeted therapy in BRAF mutant anaplastic thyroid cancer. Journal of the National Comprehensive Cancer Network 14 1203-1207. (https:// doi.org/10.6004/jnccn.2016.0130)

Baek SK, Lee MC, Hah JH, Ahn SH, Son YI, Rho YS, Chung PS, Lee YS, Koo BS, Jung KY, et al. 2016 Role of surgery in the management of anaplastic thyroid carcinoma: Korean nationwide multicenter study of 329 patients with anaplastic thyroid carcinoma, 2000 to 2012. Head \& Neck 39 133-139. (https://doi.org/10.1002/hed.24559)

Bible KC, Suman VJ, Menefee ME, Smallridge RC, Molina JR, Maples WJ, Karlin NJ, Traynor AM, Kumar P, Goh BC, et al. 2012 A multiinstitutional phase 2 trial of pazopanib monotherapy in advanced anaplastic thyroid cancer. Journal of Clinical Endocrinology and Metabolism 97 3179-3184. (https://doi.org/10.1210/jc.20121520)

Brauner E, Gunda V, Vanden Borre P, Zurakowski D, Kim YS, Dennett KV, Amin S, Freeman GJ \& Parangi S 2016 Combining BRAF inhibitor and anti PD-L1 antibody dramatically improves tumor regression and anti tumor immunity in an immunocompetent murine model of anaplastic thyroid cancer. Oncotarget 7 17194-17211. (https://doi.org/10.18632/oncotarget.7839)

Brignardello E, Palestini N, Felicetti F, Castiglione A, Piovesan A, Gallo M, Freddi M, Ricardi U, Gasparri G, Ciccone G, et al. 2014 Early surgery and survival of patients with anaplastic thyroid carcinoma: analysis of a case series referred to a single institution between 1999 and 2012. Thyroid 24 1600-1606. (https://doi. org/10.1089/thy.2014.0004)

Cabanillas ME, Williams MD, Gunn GB, Weitzman SP, Burke L, Busaidy NL, Ying AK, Yiin YH, William WN, Lu C, et al. 2017 Facilitating anaplastic thyroid cancer specialized treatment: a model
C) 2018 Society for Endocrinology Published by Bioscientifica Ltd. Printed in Great Britain 
for improving access to multidisciplinary care for patients with anaplastic thyroid cancer. Head \&Neck 39 1291-1295. (https://doi. org/10.1002/hed.24784)

Danilova L, Wang H, Sunshine J, Kaunitz GJ, Cottrell TR, Xu H, Esandrio J, Anders RA, Cope L, Pardoll DM, et al. 2016 Association of PD-1/PD-L axis expression with cytolytic activity, mutational load, and prognosis in melanoma and other solid tumors. PNAS 113 E7769-E7777. (https://doi.org/10.1073/ pnas.1607836113)

De Crevoisier R, Baudin E, Bachelot A, Leboulleux S, Travagli JP, Caillou B \& Schlumberger M 2004 Combined treatment of anaplastic thyroid carcinoma with surgery, chemotherapy, and hyperfractionated accelerated external radiotherapy. International Journal of Radiation Oncology, Biology, Physics 60 1137-1143. (https:// doi.org/10.1016/j.ijrobp.2004.05.032)

Dralle H, Musholt TJ, Schabram J, Steinmuller T, Frilling A, Simon D, Goretzki PE, Niederle B, Scheuba C, Clerici T, et al. 2013 German Association of Endocrine Surgeons practice guideline for the surgical management of malignant thyroid tumors. Langenbeck's Archives of Surgery 398 347-375. (https://doi.org/10.1007/s00423-013-1057-6)

Dumke AK, Pelz T \& Vordermark D 2014 Long-term results of radiotherapy in anaplastic thyroid cancer. Radiation Oncology 990. (https://doi.org/10.1186/1748-717X-9-90)

Godbert Y, Henriques de Figueiredo B, Bonichon F, Chibon F, Hostein I, Perot G, Dupin C, Daubech A, Belleannee G, Gros A, et al. 2015 Remarkable response to crizotinib in woman with anaplastic lymphoma kinase-rearranged anaplastic thyroid carcinoma. Journal of Clinical Oncology 33 e84-e87. (https://doi.org/10.1200/ JCO.2013.49.6596)

Goffredo P, Thomas SM, Adam MA, Sosa JA \& Roman SA 2015 Impact of timeliness of resection and thyroidectomy margin status on survival for patients with anaplastic thyroid cancer: an analysis of 335 cases. Annals of Surgical Oncology 22 4166-4174. (https://doi.org/10.1245/ s10434-015-4742-6)

Haddad RI, Lydiatt WM, Ball DW, Busaidy NL, Byrd D, Callender G, Dickson P, Duh QY, Ehya H, Haymart M, et al. 2015 Anaplastic thyroid carcinoma, version 2.2015. Journal of the National Comprehensive Cancer Network 13 1140-1150. (https://doi. org/10.6004/jnccn.2015.0139)

Haymart MR, Banerjee M, Yin H, Worden F \& Griggs JJ 2013 Marginal treatment benefit in anaplastic thyroid cancer. Cancer 119 3133-3139. (https://doi.org/10.1002/cncr.28187)

Higashiyama T, Ito Y, Hirokawa M, Fukushima M, Uruno T, Miya A, Matsuzuka F \& Miyauchi A 2010 Induction chemotherapy with weekly paclitaxel administration for anaplastic thyroid carcinoma. Thyroid 20 7-14. (https://doi.org/10.1089/thy.2009.0115)

Kunstman JW, Juhlin CC, Goh G, Brown TC, Stenman A, Healy JM, Rubinstein JC, Choi M, Kiss N, Nelson-Williams C, et al. 2015 Characterization of the mutational landscape of anaplastic thyroid cancer via whole-exome sequencing. Human Molecular Genetics 24 2318-2329. (https://doi.org/10.1093/hmg/ddu749)

Kwon J, Kim BH, Jung HW, Besic N, Sugitani I \& Wu HG 2016 The prognostic impacts of postoperative radiotherapy in the patients with resected anaplastic thyroid carcinoma: a systematic review and meta-analysis. European Journal of Cancer 59 34-45. (https://doi. org/10.1016/j.ejca.2016.02.015)

Landa I, Ibrahimpasic T, Boucai L, Sinha R, Knauf JA, Shah RH, Dogan S, Ricarte-Filho JC, Krishnamoorthy GP, Xu B, et al. 2016 Genomic and transcriptomic hallmarks of poorly differentiated and anaplastic thyroid cancers. Journal of Clinical Investigation 126 1052-1066. (https://doi.org/10.1172/JCI85271)

Latteyer S, Tiedje V, Konig K, Ting S, Heukamp LC, Meder L, Schmid KW, Fuhrer D \& Moeller LC 2016a Targeted next-generation sequencing for TP53, RAS, BRAF, ALK and NF1 mutations in anaplastic thyroid cancer. Endocrine 54 733-741. (https://doi. org/10.1007/s12020-016-1080-9)
Latteyer S, Tiedje V, Schilling B \& Fuhrer D 2016b Perspectives for immunotherapy in endocrine cancer. Endocrine-Related Cancer 23 R469-R484. (https://doi.org/10.1530/ERC-16-0169)

Lee DY, Won JK, Choi HS, Park do J, Jung KC, Sung MW, Kim KH, Hah JH \& Park YJ 2016 Recurrence and survival after gross total removal of resectable undifferentiated or poorly differentiated thyroid carcinoma. Thyroid 26 1259-1268. (https://doi.org/10.1089/ thy.2016.0147)

Lowe NM, Loughran S, Slevin NJ \& Yap BK 2014 Anaplastic thyroid cancer: the addition of systemic chemotherapy to radiotherapy led to an observed improvement in survival - a single centre experience and review of the literature. Scientific World Journal 2014674583. (https://doi.org/10.1155/2014/674583)

Marten KA \& Gudena VK 2015 Use of vemurafenib in anaplastic thyroid carcinoma: a case report. Cancer Biology and Therapy 16 1430-1433. (https://doi.org/10.1080/15384047.2015.1071734)

Mohebati A, Dilorenzo M, Palmer F, Patel SG, Pfister D, Lee N, Tuttle RM, Shaha AR, Shah JP \& Ganly I 2014 Anaplastic thyroid carcinoma: a 25-year single-institution experience. Annals of Surgical Oncology 21 1665-1670. (https://doi.org/10.1245/s10434014-3545-5)

Molinaro E, Romei C, Biagini A, Sabini E, Agate L, Mazzeo S, Materazzi G, Sellari-Franceschini S, Ribechini A, Torregrossa L, et al. 2017 Anaplastic thyroid carcinoma: from clinicopathology to genetics and advanced therapies. Nature Reviews Endocrinology 13 644-660. (https://doi.org/10.1038/nrendo.2017.76)

Orita Y, Sugitani I, Amemiya T \& Fujimoto Y 2011 Prospective application of our novel prognostic index in the treatment of anaplastic thyroid carcinoma. Surgery 150 1212-1219. (https://doi. org/10.1016/j.surg.2011.09.005)

Perros P, Boelaert K, Colley S, Evans C, Evans RM, Gerrard Ba G, Gilbert J, Harrison B, Johnson SJ, Giles TE, et al. 2014 Guidelines for the management of thyroid cancer. Clinical Endocrinology $\mathbf{8 1}$ (Supplement 1) 1-122. (https://doi.org/10.1111/cen.12515)

Pezzi TA, Mohamed ASR, Sheu T, Blanchard P, Sandulache VC, Lai SY, Cabanillas ME, Williams MD, Pezzi CM, Lu C, et al. 2017 Radiation therapy dose is associated with improved survival for unresected anaplastic thyroid carcinoma: outcomes from the National Cancer Data Base. Cancer 123 1653-1661. (https://doi.org/10.1002/ cncr.30493)

Prager GW, Koperek O, Mayerhoefer ME, Muellauer L, Wrba F, Niederle B, Zielinski CC \& Raderer M 2016 Sustained response to vemurafenib in a BRAFV600E-mutated anaplastic thyroid carcinoma patient. Thyroid 26 1515-1516. (https://doi.org/10.1089/ thy.2015.0575)

Rosove MH, Peddi PF \& Glaspy JA 2013 BRAF V600E inhibition in anaplastic thyroid cancer. New England Journal of Medicine 368 684-685. (https://doi.org/10.1056/NEJMc1215697)

Savvides P, Nagaiah G, Lavertu P, Fu P, Wright JJ, Chapman R, Wasman J, Dowlati A \& Remick SC 2013 Phase II trial of sorafenib in patients with advanced anaplastic carcinoma of the thyroid. Thyroid 23 600-604. (https://doi.org/10.1089/thy.2012.0103)

Schlumberger M, Tahara M \& Wirth LJ 2015 Lenvatinib in radioiodinerefractory thyroid cancer. New England Journal of Medicine 3721868 (https://doi.org/10.1056/NEJMc1503150)

Seto A, Sugitani I, Toda K, Kawabata K, Takahashi S \& Saotome T 2015 Chemotherapy for anaplastic thyroid cancer using docetaxel and cisplatin: report of eight cases. Surgery Today 45 221-226. (https:// doi.org/10.1007/s00595-013-0751-x)

Smallridge RC, Ain KB, Asa SL, Bible KC, Brierley JD, Burman KD, Kebebew E, Lee NY, Nikiforov YE, Rosenthal MS, et al. 2012 American Thyroid Association guidelines for management of patients with anaplastic thyroid cancer. Thyroid 22 1104-1139. (https://doi.org/10.1089/thy.2012.0302)

Sosa JA, Balkissoon J, Lu SP, Langecker P, Elisei R, Jarzab B, Bal CS Marur S, Gramza A \& Ondrey F 2012 Thyroidectomy followed by 
fosbretabulin (CA4P) combination regimen appears to suggest improvement in patient survival in anaplastic thyroid cancer. Surgery 152 1078-1087. (https://doi.org/10.1016/j.surg.2012.08.036)

Sosa JA, Elisei R, Jarzab B, Balkissoon J, Lu SP, Bal C, Marur S, Gramza A, Yosef RB, Gitlitz B, et al. 2014 Randomized safety and efficacy study of fosbretabulin with paclitaxel/carboplatin against anaplastic thyroid carcinoma. Thyroid 24 232-240. (https://doi.org/10.1089/ thy.2013.0078)

Steggink LC, van Dijk BA, Links TP \& Plukker JT 2015 Survival in anaplastic thyroid cancer in relation to pre-existing goiter: a population-based study. American Journal of Surgery 209 1013-1019. (https://doi.org/10.1016/j.amjsurg.2014.06.027)

Subbiah V, Kreitman RJ, Wainberg ZA, Cho JY, Schellens JHM, Soria JC, Wen PY, Zielinski C, Cabanillas ME, Urbanowitz G, et al. 2017 Dabrafenib and trametinib treatment in patients with locally advanced or metastatic BRAF V600-mutant anaplastic thyroid cancer. Journal of Clinical Oncology 36 7-13. (https://doi.org/10.1200/ JCO.2017.73.6785)

Sugitani I, Miyauchi A, Sugino K, Okamoto T, Yoshida A \& Suzuki S 2012 Prognostic factors and treatment outcomes for anaplastic thyroid carcinoma: ATC Research Consortium of Japan cohort study of 677 patients. World Journal of Surgery 36 1247-1254. (https://doi. org/10.1007/s00268-012-1437-z)

Sun C, Li Q, Hu Z, He J, Li C, Li G, Tao X \& Yang A 2013 Treatment and prognosis of anaplastic thyroid carcinoma: experience from a single institution in China. PLOS ONE 8 e80011. (https://doi.org/10.1371/ journal.pone.0080011)

Sun C, Li C, Hu Z, Li X, He J, Song M, Li G, Zhang F \& Li Q 2015 Influence of risk grouping on therapeutic decisions in patients with anaplastic thyroid carcinoma. European Archives of Oto-RhinoLaryngology 272 985-993. (https://doi.org/10.1007/s00405-014-2937-2)

Tahara M, Kiyota N, Yamazaki T, Chayahara N, Nakano K, Inagaki L, Toda K, Enokida T, Minami H, Imamura Y, et al. 2017 Lenvatinib for anaplastic thyroid cancer. Frontiers in Oncology 7 25. (https://doi. org/10.3389/fonc.2017.00025)

Tiedje V, Ting S, Herold T, Synoracki S, Latteyer S, Moeller LC, Zwanziger D, Stuschke M, Fuehrer D \& Schmid KW 2017 NGS based identification of mutational hotspots for targeted therapy in anaplastic thyroid carcinoma. Oncotarget 8 42613-42620.

Tiedje V, Kroiss M, Dralle H, Ting S, Stuschke M, Flentje M, Bauer S, Weber F, Brabant G, Lorenz K, et al. 2015 Protokoll zur multimodalen Therapie des anaplastischen Schilddrüsenkarzinoms. Endokrinologie Informationen 39 63-65. (https://doi.org/10.18632/ oncotarget.17300)

Wagle N, Grabiner BC, Van Allen EM, Amin-Mansour A, TaylorWeiner A, Rosenberg M, Gray N, Barletta JA, Guo Y, Swanson SJ, et al. 2014 Response and acquired resistance to everolimus in anaplastic thyroid cancer. New England Journal of Medicine 371 1426-1433. (https://doi.org/10.1056/NEJMoa1403352)

Wendler J, Kroiss M, Gast K, Kreissl MC, Allelein S, Lichtenauer U, Blaser R, Spitzweg C, Fassnacht M, Schott M, et al. 2016 Clinical presentation, treatment and outcome of anaplastic thyroid carcinoma: results of a multicenter study in Germany. European Journal of Endocrinology 175 521-529. (https://doi.org/10.1530/EJE-16-0574)

Zivaljevic V, Tausanovic K, Paunovic I, Diklic A, Kalezic N, Zoric G, Sabljak V, Vekic B, Zivic R, Marinkovic J, et al. 2014 Age as a prognostic factor in anaplastic thyroid cancer. International Journal of Endocrinology 2014 5. (https://doi.org/10.1155/2014/240513)

Received in final form 19 December 2017

Accepted 2 January 2018

Accepted Preprint published online 2 January 2018 (c) 2018 Society for Endocrinology Published by Bioscientifica Ltd. Printed in Great Britain 